\title{
L'Amérique ou le grand renoncement à Durkheim
}

\section{Emmanuel Désveaux}

\section{OpenEdition}

\section{Journals}

Édition électronique

URL : https://journals.openedition.org/jsa/2916

DOI : 10.4000/jsa.2916

ISSN : 1957-7842

\section{Éditeur}

Société des américanistes

\section{Édition imprimée}

Date de publication : 5 janvier 2005

Pagination : 17-79

ISSN : 0037-9174

\section{Référence électronique}

Emmanuel Désveaux, "L'Amérique ou le grand renoncement à Durkheim », Journal de la Société des américanistes [En ligne], 91-1 | 2005, mis en ligne le 04 octobre 2006, consulté le 03 septembre 2022 URL : http://journals.openedition.org/jsa/2916 ; DOI : https://doi.org/10.4000/jsa.2916 


\title{
POSITION
}

\section{L'AMÉRIQUE OU LE GRAND RENONCEMENT À DURKHEIM}

\author{
Emmanuel DÉSVEAUX *
}

Quadratura americana, essai d'anthropologie lévi-straussienne a fait l'objet d'un commentaire brillant, fouillé, précieux de la part de Klaus Hamberger dans la précédente livraison du Journal de la Société des Américanistes '. J'en suis heureux et flatté, d'autant plus que ce texte fait suite à un autre de même qualité dû à la plume de Lucien Scubla et paru en 2003 dans L'Homme ${ }^{2}$. Les lectures exigeantes de Hamberger et de Scubla me font honneur comme elles créent les conditions d'une vraie discussion autour de mes thèses. Par ailleurs, dans plusieurs autres revues professionnelles, le livre a fait l'objet d'une recension ${ }^{3}$. Il me semble utile de les verser au dossier en dépit de leur moindre développement car toutes sont à un titre ou à un autre significatives de la réception de mon travail dans la communauté scientifique. Cela nous permet d'élaborer une plate-forme de débat élargie, profitable à tous.

Toutefois en guise de viatique pour le lecteur néophyte à la discussion, je débuterai par un résumé, aussi bref que possible, du livre en question. Il a pour socle théorique Les Mythologiques de Lévi-Strauss, autrement dit l'unité de l'Amérique en vertu d'un vaste système de transformations des mythologies locales les unes dans les autres. Quadratura americana comprend quatre parties. La première tente de montrer que la logique transformationnelle n'est pas circonscrite au mythe. Elle touche tout aussi bien le domaine du rituel que des éléments de la culture matérielle. Elle s'articule autour d'une relecture du mythe du dénicheur d'oiseaux qui sert de référence à Lévi-Strauss dans sa tétralogie en montrant son enracinement profond dans l'ordre du rituel et en dégageant sa portée sociologique. Cette section comporte également une discussion relative aux quadrants comme instruments d'analyse formelle. Sont ainsi envisagées les relations qu'entretiennent le groupe de Klein et la formule canonique des mythes

* EHESS et Musée du quai Branly, Paris [emmanuel.desveaux@ehess.fr].

Journal de la Société des Américanistes, 2005, 91-1, pp. 71-79. (O Société des Américanistes. 
inventée par Lévi-Strauss, lesquels seront utilisés à plusieurs reprises dans la suite du livre. La seconde partie tente de tirer les conséquences de cette extension de la qualité transformationnelle du matériau ethnographique amérindien sur le plan de l'ontologie. Une de ses propositions les plus marquantes réside dans une réflexion sur l'essence de la guerre amérindienne, synonyme d'une négation de l'altérité. La troisième partie, qui introduit la notion de "socièmes élémentaires », cherche à illustrer la valeur heuristique de l'approche transformationnelle afin de résoudre certaines diffícultés locales, tel le cas des soi-disant castes natchez ou celui de la réincarnation inuit, que pose de façon classique l'ethnographie nord-américaniste. La dernière partie s'attaque au thème de la parenté. Elle suggère que les nomenclatures nord-américaines entretiennent un rapport transformationnel entre elles. Celles-ci ne reflètent pas des règles de mariage qui, elles-mêmes, traduiraient la variabilité d'un échange matrimonial posé comme un universel. Entrant en résonance avec certaines considérations finales de la première partie quant à la filiation, cette section se propose enfin d'en donner une signification à l'échelle du continent qui soit radicalement différente de celle généralement admise dans notre discipline. Tirant les conséquences de l'absence d'échange matrimonial en Amérique - ou plutôt de sa non-généralité -, une très brève conclusion s'attache à définir un contenu à la prohibition de l'inceste dans le contexte de ce que l'on pourrait appeler la méga-aire culturelle amérindienne.

Nul doute que le programme de ce livre fut ambitieux. Mais il ne prétend à rien d'autre qu'au statut d'essai comme son sous-titre l'indique et comme en conviennent nombre de recenseurs. En réalité, son ambition coïncide avec l'ampleur de son objet dès lors que nous souhaitions montrer la pertinence de l'analyse transformationnelle au niveau du rituel, de la technique, de la sociologie et des nomenclatures de parenté, autrement dit de tout ce qui, à l'exclusion néanmoins de la langue, constitue une culture telle que l'entendent communément les anthropologues boasiens américains. L'ambition inspire tantôt l'agacement, tantôt l'admiration, tantôt la distanciation plus ou moins empreinte d'ironie. On retrouve ces trois sentiments chez les recenseurs de Quadratura americana. Il n'y aurait guère lieu d'insister sur ce point sinon pour souligner à cette occasion une sorte de distorsion entre le fond et la forme, phénomène qui n'a rien d'exceptionnel et qui contribue souvent au charme de la recension comme genre littéraire. Car admiration, agacement ou ironie, laquelle est susceptible d'être plus ou moins froide, appartiennent à la forme. Or certaines recensions sont chaleureuses dans la forme, tout en étant assez, voire résolument critiques sur le fond, tandis que d'autres manifestent à l'évidence une adhérence, serait-elle partielle, aux thèses exposées ou, à tout le moins, un réel intérêt pour elles, tout en restant extraordinairement froides, sinon glaciales. Ces considérations générales sur la tonalité de l'écriture me conduisent à en formuler immédiatement une autre touchant un point beaucoup plus précis, mais qui relève toujours selon moi de la forme. À travers plusieurs textes, on sent un reproche larvé. Chez Müller, il a le 
mérite de s'exprimer ouvertement. J'aurais manqué de respect à la personne de Claude Lévi-Strauss, d'une part, en m'étant approprié son nom dans le sous-titre, d'autre part, en ayant contesté une de ses idées maîtresses, en l'occurrence l'échange matrimonial... et tout cela sans son autorisation. Il me semble que le procès n'a pas lieu d'être. Lévi-Strauss est désormais un auteur classique, à l'instar d'un Mauss ou pour remonter plus haut dans le temps d'un Durkheim ou encore, pour élargir aux traditions parallèles à la nôtre, d'un Boas et d'un Malinowski. Il ne l'est pas pour des motifs institutionnels. Il l'est devenu au fil du temps grâce à la puissance de sa pensée. Remarquons qu'il y a moins d'écart de temps entre la rédaction des grands textes de Mauss qui scande la première moitié $\mathrm{du} \mathrm{xx}^{\mathrm{e}}$ siècle et celle des Structures élémentaires de la parenté, qu'entre la parution de ce dernier livre, voire de La Pensée sauvage, et aujourd'hui. Or le propre d'un auteur classique est de n'appartenir à personne, ni à aucune école, même si une ou plusieurs écoles peuvent se prévaloir de lui. Il est susceptible d'être discuté par tous, dans n'importe quel terme pour autant que l'on reste dans le cadre de la raison académique. L'emploi des adjectifs "marxiste », "durkheimien » ou « maussien » ne suscite ainsi aucune diffículté, en ce qu'ils signalent des champs de réflexion spécifiques, assis sur la pensée d'un auteur, même si leur contenu ne fait pas l'unanimité. En tout état de cause, la critique argumentée à l'égard de l'auteur de référence a pleinement droit de cité. Elle se situe même au cœur de notre activité scientifique. Pour reprendre l'exemple de Mauss, ce n'est pas faire injure à son œuvre, ni être foncièrement anti-maussien, que de noter le décalage entre l'auteur de l'Essai sur le don, qui semble valoriser d'un point de vue moral les institutions primitives, et celui de l'Évolution de la notion de personne, qui apparaît plus ouvert aux sirènes de l'évolutionnisme. Claude Lévi-Strauss n'abuse aucunement de son statut d'auteur majeur. On peut s'étonner dès lors du zèle de certains dévots à défendre une orthodoxie lévi-straussienne que l'auteur d'Anthropologie structurale n'a jamais promue personnellement, ce qui ne l'a nullement empêché de rappeler à l'occasion ses positions, ainsi qu'il l'a encore fait récemment à propos de l'échange matrimonial ${ }^{4}$.

Abordons maintenant le fond des choses et commençons, pour ce faire, par considérer la question de la formalisation, en particulier le recours aux quadrants. Celle-là m'attire des remarques graduées. Il y a le rejet pur et simple comme chez Lézé, afin de mieux disqualifier l'ensemble du livre et, à travers lui, toute anthropologie autre que postmoderniste. La véritable question est plutôt de savoir dans quelles conditions une formalisation procure un bénéfice heuristique, compte tenu de l'inévitable construction des données qu'elle implique par retranchement de leur contexte. C'est bien du moins en ces termes que s'interroge Arcand à propos de Quadratura Americana tout en soulignant que le problème se posait exactement dans les mêmes termes pour Lévi-Strauss lorsqu'il rédigeait les Mythologiques. Ensuite, on a pu me reprocher d'avoir utilisé intensivement la formule canonique des mythes en opposition à Lévi-Strauss qui l'aurait fait avec 
parcimonie. Là encore, le procès nous apparaît pour le moins singulier. D'abord parce que, si la formule est absente des Mythologiques, elle revient bel et bien en force dans La Potière jalouse et dans Histoire de lynx, que l'on peut considérer comme deux textes ayant la plus forte valeur testamentaire de toute l'œuvre lévi-straussienne ; ensuite parce que revendiquer un héritage intellectuel, et donc emprunter la fameuse formule - qui s'avère en outre être un objet théorique doté d'une autonomie relativement grande -, n'implique pas d'imiter son inventeur en tout. Hamberger adopte finalement une stratégie mitoyenne à celle de Lézé, quoique sur un mode mineur. S'il concède l'utilité d'une formalisation à base de quadrants, il s'attaque vivement à la formule canonique des mythes et à l'usage que nous en avons fait. Il le fait dans la section terminale de son texte, ce qui lui permet d'instiller, dans les considérations conclusives qui prennent nécessairement sa suite, un soupçon quant à la pertinence globale de notre travail.

Le thème de la formalisation se situe à mi-chemin de la forme et du fond. Concentrons-nous sur ce dernier désormais. Dans l'ensemble, l'idée d'étendre, à l'encontre de Lévi-Strauss, l'analyse transformationnelle au rituel plait. Scubla se montre même enthousiaste à cet égard, non sans toutefois tirer un peu trop la couverture à lui, se faisant le héraut du primat sacrificiel. Soyons clair sur ce léger différend : sij'inscris parfois dans un même axe transformationnel des séquences rituelles « banales » et d'autres décrites de façon classique comme des sacrifices, ce n'est certainement pas pour généraliser le sacrifice dans une quelconque perspective girardienne, mais bien plutôt pour montrer sa non-spécificité en tant qu'acte rituel $^{5}$. De même, on me crédite volontiers également de la découverte que cette logique transformationnelle s'applique aux éléments de la culture matérielle.

Le statut du dualisme hante depuis longtemps la discipline. Il est sous-jacent à Quadratura Americana ainsi que Scubla et Hamberger le soulignent avec justesse. S'agit-il d'une traduction dans l'organisation sociale d'un principe dichotomique fondamental, le binarisme, ou, plutôt, d'un effet dérivé, en l'occurrence la répercussion à ce niveau-là d'une division à vocation matrimoniale ? On sait que Lévi-Strauss défend la deuxième option, notamment dans un article célèbre écrit en hommage à Josselin de Jong ${ }^{6}$ et qui prenait appui sur des cas ethnographiques amérindiens et indonésiens. Ma position est inverse, ce à quoi Scubla applaudit, en particulier parce que cela affaiblit le dogme de l'universalité de l'échange matrimonial à laquelle, lui aussi, ne semble guère croire. Mais il refuse de me suivre lorsque j'essaye de démontrer qu'en Amérique la guerre n'est pas un mécanisme de reconnaissance de l'altérité, mais la production, conforme d'une part à une logique de prédation, d'autre part à des autonymies qui postulent toujours l'exclusivité, d'un néant périphérique qui a pour effet d'attribuer la qualité d'humanité aux seuls membres du groupe. Scubla en appelle alors au principe de réciprocité, celui-là même qu'il envisageait fort opportunément de dénoncer quelques lignes auparavant en matière de parenté. Certes, ici, la réciprocité serait d'ordre rituel (autrement dit, chez Scubla, d'ordre quasi- 
métaphysique) et, en Amérique, si les groupes se livrent en permanence à la guerre, c'est qu'ils sont en réalité dans une relation de réciprocité en s'échangeant continuellement des morts entre eux. J'avoue que cette thèse, sorte de sens commun de notre discipline, me laisse de plus en plus perplexe. La pensée anthropologique moderne a été le lieu d'une curieuse dérive, dont le point de départ réside dans la réciprocité maussienne. Le célèbre schéma concentrique de Sahlins illustre parfaitement le phénomène ${ }^{7}$. Toute la démonstration de l'auteur de l'Essai sur le don repose sur la matérialité de l'objet donné. On donne à l'autre un élément qu'il gardera par-devers lui jusqu'à l'échéance du contre-don. C'est en définitive bien dans la nature " gagée » de la chose donnée ${ }^{8}$ que gît l'établissement en puissance d'un lien social. Cette conception s'accorde sans difficulté avec l'extension que Lévi-Strauss propose de la théorie maussienne aux relations entre unités sociales dans le cadre du mariage. En revanche, son extension à la guerre apparaît beaucoup plus problématique. Car, lorsque la violence externe entraîne la mort d'un ennemi, elle n'entraîne aucun transfert d'un groupe à un autre. La mort revient simplement à une perte, à une destruction pour le groupe dont l'un des guerriers meurt sous les coups ennemis. Rien, ni personne, n'est susceptible d'être "gagé » auprès d'un éventuel autrui, puisque nous sommes désormais dans le creux, l'absence, le manque ; la mort débouche sur une dématérialisation radicale, sinon immédiate, du moins à très court terme. Certes, on pourrait argumenter que la prise de trophées (des scalps, des têtes réduites ou non, parfois un simple membre) relève d'un commerce matériel. Mais, en Amérique du moins, cette position est intenable pour deux raisons. Premièrement, toutes les ethnographies concordent pour dire que les trophées ont une dimension essentiellement symbolique, ce que signale d'ailleurs l'existence récurrente de procédures rituelles de dé-substantivation de l'élément prélevé. Deuxièmement, le trophée sert uniquement, si l'on peut dire, la cause du guerrier vainqueur. Il ne fournit aucun gage ; au contraire, il renvoie le vaincu dans les limbes du néant, quand bien même il y a accaparement de son nom ou de sa personne par le guerrier vainqueur. Car, en l'espèce, il ne fait que s'approprier l'ombre d'une personne réelle, un fragment issu de son démantèlement. En bref, la mort guerrière va à l'encontre de la nécessité, inhérente à la perspective d'un contre-don, d'une permanence de l'être. Ce n'est pas parce que cette mort suscite un sentiment de vengeance qu'elle détermine une réciprocité, du moins entendue dans le sens maussien qui prévaut dans notre discipline. Et d'ailleurs, est-ce de la vengeance ou, plutôt, un impératif absolu de faire la guerre? Nous retraiterons de ce point dans la mesure où un examen plus attentif de la façon dont Hamberger présente mes thèses va nous conduire à y revenir.

Hamberger offre une très belle synthèse de ce qui se joue au cœur de Quadratura americana, réussissant, peut-être mieux que je n'ai su le faire moi-même, à établir le pont entre la dimension mythico-rituelle et celle strictement sociologique de mon argument. J'aimerais seulement attirer l'attention du lecteur de son 
texte sur deux points, en apparence mineurs, mais qui, combinés, sont susceptibles de biaiser la compréhension de mes intentions. Discutant de nos socièmes élémentaires, à savoir respectivement, (a) le groupe de filiation (plus ou moins équivalant à un clan), (b) l'entité endogame, (c) le groupe résidentiel et, enfin, (d) la confrérie guerrière ou la société secrète, il me reproche de ne pas définir les groupes de filiation et de résidence, «ce qui nourrit le soupçon qu'il les définit justement par l'absence ou, du moins, par l'insignifiance des liens consanguins et des mariages locaux [...] » (p. 108). Rien n'est plus faux que cela précisément. Selon les circonstances, autrement dit selon les différentes sociétés, mais aussi selon les différentes saisons ${ }^{9}$, l'organisation sociale observable relèvera d'une combinaison - qui vaut partiellement recouvrement - de deux socièmes élémentaires, voire de trois, mais jamais des quatre. Les socièmes élémentaires ne sont pas des entités au statut pseudo-juridique, à l'instar des « principes d'organisation sociale » des manuels classiques d'anthropologie. Ce sont des entités d'ordre sémantique, translation dans le registre du sociologique, autrement dit d'un collectif, des quatre temporalités primordiales qui caractérisent l'existence individuelle : naître (ce qui veut dire avoir des aînés ou des cadets), vivre au quotidien (ce qui implique de se procurer de la nourriture, soit en la " produisant » soimême, soit en la recevant par partage), "s'affininiser » par mariage et, enfin, affronter la mort. Un tel fondement sémantique du sociologique apparaît inadmissible à Hamberger. Pour preuve, la citation suivante : « l'argument [de Désveaux] devient circulaire. En effet, on peut difficilement se défendre de l'impression qu'il a été développé à l'extérieur de l'analyse comparative des organisations sociales et tiendrait moins de la sociologie que de l'idéologie des Amérindiens, telle qu'elle ressort dans l'analyse des mythes ». L'argument n'a rien de circulaire. Mais il est vrai qu'il revient à refuser la vieille dichotomie (hégélienne, marxiste ou, plus près de nous, lévi-straussienne) ${ }^{10}$ qui veut qu'il y ait, d'une part, le domaine du concret - du côté duquel se rangeraient les modalités régissant les organisations sociales -, d'autre part, le monde des idées ou des représentations. Autrement dit, l'infrastructure et la superstructure. Or c'est précisément avec cette tradition que nous souhaitons faire rupture en essayant de montrer que les Amérindiens vivent bien selon leurs mythes ou, encore, que leurs mythes correspondent bien à leur mode de vie. Leurs mythes ne sont pas un simple reflet imparfait du monde réel, ni même, comme l'écrit Lévi-Strauss dans La Potière jalouse, un dispositif visant à résoudre les contradictions du réel ${ }^{11}$.

La transitivité absolue du système transformationnel explique, selon nous, l'apparent paradoxe que constituent la grande diversité entre cultures locales, générée par une logique transformationnelle fondée sur l'inversion, et l'espèce d'exclusivité ontologique qui les caractérise toutes. Si chaque culture locale nie aux autres son humanité - d'où la guerre perpétuelle et l'absence d'altérité -, ce système permet à chaque individu, à chaque intelligence amérindienne, notamment quand il est fait prisonnier et adopté, d'adhérer d'emblée à ce qu'il a refusé 
même de reconnaître jusqu'alors. Grâce à cette réversibilité des points de vue, on se retrouve de plain-pied avec le perspectivisme qui jouit à l'heure actuelle des faveurs des cercles américanistes. La somme pratiquement finie de ces points de vue nous autorise néanmoins à parler, à l'instar d'Hamberger, de monades pour rendre compte de cette situation. Sauf que lui parle de monades fermées (« sans fenêtres », p. 112). Un peu plus haut, il affïrme que, selon cette même conception, les cultures amérindiennes ne pourraient apprendre «ni [du] passé, ni [de] l'Autre » (p. 109). Je laisse à Hamberger le soin de nous éclairer sur l'identité de cet autre muni de son grand $A$. Plus sérieusement, il me semble que rien n'est plus faux par rapport à notre vision des choses. La monadologie américaniste que nous prônons se caractérise au contraire par sa grande ouverture... sur la totalité d'un continent qui s'étend sur des millions de kilomètres carrés, qui possède pratiquement tous les types d'environnement naturel répertoriés et dont les cultures résultent d'une histoire - ou d'une évolution, ici le choix des mots m'importe assez peu - ayant plusieurs millénaires de profondeur. Les événements qu'ont connus ces groupes humains, les guerres, les adoptions, les rencontres, les inventions techniques, les intrusions dans de nouveaux espaces, comptent comme autant d'expériences qu'ils ont intériorisées et qui les ont modifiés au fil du temps. L'incroyable richesse des cultures amérindiennes que l'ethnologie, l'histoire ou l'archéologie nous donnent à connaître, résulte précisément de ces processus. Ce que nous disons simplement, c'est que ces changements se sont opérés en vertu du système transformationnel, lequel engendre des distinctions culturelles locales qui demeurent dans un rapport d'homothétie entre elles. C'est là que réside, selon nous, la spécificité de l'Amérique en tant qu'aire culturelle. Nous sommes conscients également que les règles ont changé depuis cinq siècles, à savoir depuis que les Amérindiens sont soumis à la pression d'une autre façon de faire (et pas seulement d'écrire) l'histoire. Mais là n'était pas le sujet de mon livre ${ }^{12}$.

En bref, ce qui ne « passe » pas dans notre propos, ni chez Scubla, ni chez Hamberger, ni vraisemblablement encore chez de nombreux collègues, renvoie à un double renoncement auquel ils ne sont manifestement pas disposés. Renoncement d'abord à une conception substantiviste, durkheimienne, du sociologique, au bénéfice d'une conception purement sémantique. L'état de guerre permanent vis-à-vis de son extérieur qui caractérise l'éthos amérindien signifie la nonaltérité ; elle ne l'instaure nullement. Renoncement ensuite à l'universalité, au profit d'une analyse s'attachant à une aire culturelle restreinte, serait-elle de la taille d'un contiment. Abstraction faite de l'échelle, Quadratura americana ne serait-il pas, en définitive, un texte plus boasien que lévi-straussien ? Si, à l'évidence, je ne m'en étais pas aperçu lorsque j'intitulais mon livre, la lecture de mes critiques parisiens a commencé à m'en convaincre. 


\section{Une ultime remarque}

Dans la conclusion de son commentaire, Hamberger suggère de passer à une nouvelle étape de l'anthropologie transformationnelle en faisant appel à des procédures informatisées. Il m'est arrivé moi-même de rêver par le passé à une démonstration validée par ordinateur. Mais je dois avouer nourrir un scepticisme de plus en plus aigu à ce sujet. Et ce, pour deux raisons principales. La première tient au problème de la codification. Contrairement à ce qu'affirme Hamberger, je ne crois pas à des progrès sensibles sur ce plan depuis l'époque où Lévi-Strauss émettait, pour la première fois, l'idée d'avoir recours à des ordinateurs pour résoudre certains problèmes de notre discipline. Les seuls progrès - qui sont loin d'être négligeables car, en tout état de cause, une telle entreprise supposerait la manipulation de corpus considérables -, concernent la puissance de calcul des machines. La codification des données de l'ethnographie reste un redoutable défi. La seconde raison est de nature heuristique. Jusqu'à nouvel ordre, les ordinateurs ne font que répondre aux questions dont on leur a déjà appris la réponse. Ils ne font le plus souvent que conforter des idées reçues. Cela explique pourquoi notre époque les chérit tant.

\section{NOTES}

1. Sous le titre "Le continent logique, à propos de Quadratura Americana d'Emmanuel Désveaux », Journal de la Société des Américanistes, 90-2, 2004, pp. 103-114.

2. Sous le titre « Le Structuralisme et ses transformations: des Mythologiques aux logiques du rite ", L'Homme, 167-168, 2003, pp. 297-306.

3. Soit, par ordre de parution, Samuel Lézé, Anthropologie et Sociétés, 26 (2-3), 2002, pp. 266-267; Jean-Claude Müller, Recherches amérindiemmes au Québec, XXXII (2), 2003, pp. 133-135; Bernard Arcand, Current Anthropology, 45 (3), June 2004, pp. 429-430 (sous le titre " The Americas : perhaps square but never boring ") ; Carlos Fausto, Journal of The Royal Anthropological Institute (incorporating Man), 10 (4), 2004, pp. 916-917.

4. Voir " Postface ", L'Homme, 154-155, 2000, pp. 713-720.

5. Dans sa fougue, Scubla remodèle un peu trop à sa convenance l'ethnographie que je mobilise. Par exemple, rien dans mon texte (ni dans les sources que j'avais consultées) ne laisse croire que la jeune fille blackfoot qui, lors d'un rituel du type tente tremblante, invoque l'esprit de son père ou de son frère disparu récemment serait par ailleurs tenue pour responsable de son décès.

6. "Les organisations dualistes existent-elles? », Anthropologie structurale, Paris, Plon, 1958, chap. VIII.

7. Voir Marshall Sahlins, Âge de pierre, âge d'abondance, l'économie des sociétés primitives, Paris, Gallimard, 1976 [1972], p. 253.

8. La relecture de Mauss à laquelle Maurice Godelier se livre abonde dans notre sens. Les éléments qu'une société conserve en opposition à ceux qu'elle met en circulation ne se définissent que par leur matérialité, n'ayant souvent ni forme, ni fonction précise. Notons au passage que les matériaux ethnographiques qui se situent à l'origine de toute cette discussion, chez Mauss comme chez Godelier, comme d'ailleurs chez Sahlins, sont invariablement d'origine océanienne. Voir Maurice Godelier, L'Énigme du don, Paris Fayard, 1996. 
9. En effet, afin certes d'en généraliser la leçon à l'ensemble des sociétés amérindiennes, nous nous étions fait, ici, un lecteur très fidèle du Mauss de l'" Essai sur les variations saisonnières des sociétés eskimos " qui mettait en avant l'hypothèse qu'en fonction des saisons les Inuit possèdaient des organisations complètement distinctes.

10. Voir, entre autres références, Claude Lévi-Strauss et Didier Éribon, De près comme de loin, Paris, Plon, 1988, pp. 151-152.

11. Voir La Potière jalouse, Paris, Plon, 1985, pp. 227-228.

12. Carlos Fausto voit dans le fait que Quadratura americana se focalise sur l'Amérique du Nord une limite à la pertinence de notre propos. À cela, on répondra d'abord que plusieurs passages du livre font directement appel à des données ethnographiques provenant des basses terres sud-américaines. Mais, surtout, on ajoutera que l'histoire des cinq derniers siècles a probablement moins bouleversé le paysage ethnographique nord-américain que sud-américain, rendant beaucoup plus lisibles les grands glacis transformationnels qui s'étendent d'une extrémité à l'autre du sous-continent. Pratiquement tous les spécialistes s'accordent en effet pour dire aujourd'hui que l'Amazonie a souffert d'une dépopulation brutale à la suite de l'arrivée des Européens. Il en a résulté un repeuplement autochtone et une recomposition des identités ethniques locales qui ne répond pas forcément à la dynamique " normale » du système transformationnel panaméricain. 